\title{
Controlling the Chemical Composition of Hydroxyapatite Thin Films using Pulsed Laser Deposition
}

\author{
Hiroaki Nishikawa* and Ryota Yoshikawa \\ Faculty of Biology-Oriented Science and Technology, Kinki University, 930 Nishi-Mitani, Kinokawa, Wakayama \\ 649-6493, Japan \\ * Corresponding author: Fax: 81-736-77-4754, e-mail: nishik32@waka.kindai.ac.jp
}

To prepare hydroxyapatite $\left(\mathrm{Ca}_{10}\left(\mathrm{PO}_{4}\right)_{6}(\mathrm{OH})_{2}\right)$ thin films with a stoichiometric $\mathrm{Ca} / \mathrm{P}$ ratio via pulsed laser deposition (PLD), the spot area of the laser pulse was changed independently of the laser fluence. To maintain a constant fluence when the spot area was changed, the total energy irradiated on the target surface was adjusted relative to the spot area. We found that the spot area dramatically changed the $\mathrm{Ca} / \mathrm{P}$ ratio of the hydroxyapatite thin films that were prepared via PLD with a constant fluence. The best $\mathrm{Ca} / \mathrm{P}$ ratio of $1.72 \pm 0.13$, which is close to the stoichiometric ratio (1.67), is achieved with a spot area and a fluence of $3.8 \mathrm{~mm}^{2}$ and $2.4 \mathrm{~J} / \mathrm{cm}^{2}$, respectively. We report on the importance of the spot area in the PLD technique for the first time.

Key words: pulsed laser deposition, hydroxyapatite, $\mathrm{Ca} / \mathrm{P}$ ratio, laser pulse spot area, laser fluence

\section{INTRODUCTION}

Hydroxyapatite (HA), $\mathrm{Ca}_{10}\left(\mathrm{PO}_{4}\right)_{6}(\mathrm{OH})_{2}$, is a main component of bones and teeth. HA is widely applied in medical and dental devices ${ }^{1-5}$ as a useful bioceramic because of its excellent biocompatibilities that result from its bone conduction property. One of the most general applications of HA is not implantation of HA alone into a living body, but rather coating of a thin layer of HA on the surfaces of metallic medical and dental devices. This composite material is necessary because HA as an ionic crystal has poor mechanical toughness. Reducing the thickness of the coated layer mitigates this drawback of HA. A major advantage of $\mathrm{HA}$ as a bioceramic is that cells on the HA surface are able to react because of the excellent biocompatibilities of HA. It is sufficient that the surfaces of the medical and dental devices are covered with HA; the material of the inner devices does not necessarily need to be made from HA. In terms of using HA as a surface coating material, it is of paramount importance that HA is coated on the surfaces of the medical and dental implants as a high-quality, thin, high-density, high-adhesion strength layer. To date, many coating techniques have been examined, including plasma spraying ${ }^{2}, 3,6,7$, ion beam sputtering ${ }^{8-10}$, magnetron sputtering $^{11,12}$ and pulsed laser deposition (PLD) ${ }^{13,14}$. Among these techniques, the plasma spraying technique is inferior because the thickness of the plasma-sprayed HA layer is at least several tens of $\mu \mathrm{m}^{15}$; the technique is most widely used for commercial production of medical and dental devices because of its high growth rate. The plasma spraying technique has another drawback in that the chemical composition of HA deviates from the stoichiometric $\mathrm{Ca} / \mathrm{P}$ ratio of $10 / 6 \approx 1.67$, because of the evaporation of the relatively volatile phosphorus ${ }^{16}$. The stoichiometry of the HA thin films is quite important to ensure fabrication of high-quality medical and dental devices, because non-stoichiometric HA exhibits pronounced biodegradability ${ }^{17}$, and these HA coatings quickly disappear.

To date, we have demonstrated that the PLD technique can be used to deposit very high-quality HA thin films that are characterized by high density and high adhesion strength ${ }^{14,18-21}$. One of the greatest advantages of the PLD technique is that the grown thin film has a similar chemical composition to the source of the thin film (i.e., the so-called target). However, the HA thin films that we grew previously deviated from the stoichiometric chemical composition. Energy-dispersive X-ray (EDX) analysis showed ${ }^{21}$ that our HA thin films had a $\mathrm{Ca} / \mathrm{P}$ ratio $>2.0$. To improve the chemical composition of these HA thin films, we have already reported $^{21}$ that the atmospheric pressure of the $\mathrm{O}_{2}+\mathrm{H}_{2} \mathrm{O}$ gas mixture used during $\mathrm{HA}$ growth can move the $\mathrm{Ca} / \mathrm{P}$ ratio toward the stoichiometric value of 1.67. However, pressure is one of the most important parameters for growth of the appropriate material according to the phase rule. If the chemical composition of HA can be controlled by other parameters independently of the pressure and of any other important parameters with regard to the phase rule, it is better to grow high-quality HA thin films by varying these parameters rather than the pressure. To develop a new way to control the chemical composition of HA by PLD, we have studied the spot area of the laser pulse on the target during PLD, as already reported in the literature 22 . 23. In these previous studies ${ }^{22,23}$, however, the laser fluence, i.e., the energy density of the laser (where the total energy is divided by spot area), also changed with the spot area because the authors changed only the spot area and kept the total energy constant. As a result, the effects of spot area could not be evaluated exactly. In general, the fluence is recognized as one of the most critical parameters in ablation processes ${ }^{22,23}$. As a result, we chose to investigate the dependence of the $\mathrm{Ca} / \mathrm{P}$ ratio on the spot area using the PLD technique while assuming a constant fluence. 


\section{EXPERIMENT}

The HA thin film was deposited on a pure Ti substrate disk (Sumitomo Metals Naoetsu, Joetsu, Japan, purity $\geq$ $99.427 \%$ ) with a diameter and thickness of 15 and 0.5 $\mathrm{mm}$, respectively. First, the surface was manually polished using silicon carbide papers with grades ranging from $\# 80$ to $\# 4000$. Next, the surface was polished using $\mathrm{Al}_{2} \mathrm{O}_{3}$ powders with diameters ranging from 3 to $0.05 \mathrm{~mm}$ on a motor-driven rotating stage. We then used PLD to grow the HA thin film in a growth chamber at a base pressure of $10^{-4} \mathrm{~Pa}$. A KrF excimer laser (Lambda Physik, Göttingen, Germany, COMPex $102, \lambda=248 \mathrm{~nm}$ ) was used and the laser pulse repetition rate was $10 \mathrm{~Hz}$. The target ablated by the laser was commercially available pure HA pellets (HOYA Technosurgical, Tokyo, Japan, Pentax CELLYARD ${ }^{\mathrm{TM}}$, purity $\geq 99 \%$ ). The distance between the target and the Ti substrate was $38 \mathrm{~mm}$. The Ti substrate was kept at room temperature during $\mathrm{HA}$ thin film growth. $\mathrm{An}_{2}+$ $\mathrm{H}_{2} \mathrm{O}$ gas mixture was generated by bubbling pure $\mathrm{O}_{2}$ gas though a distilled water bath and the gas mixture was introduced into the growth chamber at a pressure of 0.1 $\mathrm{Pa}$.

The spot area was simply controlled by changing the distance between the target and the focal lens, as shown in Fig. 1. After the lens position was fixed, the target was pre-ablated and the two sides of the dent area, i.e., the spot area, were measured using vernier calipers. Next, the HA thin film was deposited at the same lens position as that used for the pre-ablation. The spot area values used in the study were $0.77,1.6,3.8,4.1$ and 4.7 $\mathrm{mm}^{2}$. The laser energy was measured using an energy meter (Coherent, Santa Clara, CA, USA, J-50MUV-248, $7.365 \mathrm{~V} / \mathrm{J}$ ) placed in front of the focal lens. The laser passed through a synthetic quartz window into the growth chamber with a transmittance of 0.9. The laser energy was adjusted to ensure that the values of the fluence $F$ calculated using the equation $F=[0.9 \times$ (laser energy)/(spot area)] were $1.8 \mathrm{~J} / \mathrm{cm}^{2}$ and $2.4 \mathrm{~J} / \mathrm{cm}^{2}$. In this study, the spatial distribution of the laser energy was not homogenized. Because the laser energy consistently shows a similar Gaussian distribution profile in this situation, regardless of the spot area, $F$ means the spatial average of the energy density.

The thickness and the $\mathrm{Ca} / \mathrm{P}$ ratio of the prepared HA thin films were measured using a stylus surface profiler (Veeco, Plainview, NY, USA, Dektak 150) and by EDX spectroscopy (EDAX, Mahwah, NJ, USA, Genesis combined with Elionix, Hachioji, Japan, ERA-8800, with electron beam acceleration voltage of $15 \mathrm{kV}$ ).

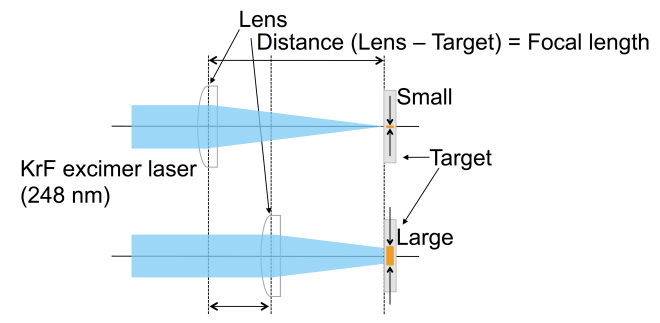

Fig. 1. Spot area can be controlled by changing the position of the focal lens.
These measurements were performed for five points with spacing of $2 \mathrm{~mm}$ to evaluate the spatial distributions of the thickness and the $\mathrm{Ca} / \mathrm{P}$ ratio; the data were averaged for three different samples for each spot area. All HA thin films used in this study had a maximum thickness of $250 \pm 25 \mathrm{~nm}$.

\section{RESULTS AND DISCUSSION}

Figure 2 shows the variation of the spatial distribution of the thickness for (a) $F=1.8 \mathrm{~J} / \mathrm{cm}^{2}$ and (b) $F=2.4$ $\mathrm{J} / \mathrm{cm}^{2}$. In this figure, the vertical axis is normalized with respect to the highest thickness of each sample. We clearly see that the fluence does not dramatically affect the spatial distribution of the thickness; however, larger spot area values are clearly correlated with a larger spatial distribution. These results can be explained as follows. A large spot area is considered to be composed of a set of small spot areas that can be treated as point sources of the chemical species during the ablation process. Each small spot area desorbs the chemical species with a three-dimensional distribution with an expansion process that is oriented toward the substrate. For the larger spot area, which is a set of small spot areas, the density of the desorbed chemical species at each position is added to the chemical species from each small spot area. In this simple model, the density normal to the target surface is high because of the accumulation of the desorbed chemical species from each small spot area when compared with directions away from normal. We can explain accordingly how the spatial distribution of the thickness narrows for larger spot areas.

Figure 3 shows the spatial variation of the $\mathrm{Ca} / \mathrm{P}$ ratio for various spot area values for (a) $F=1.8 \mathrm{~J} / \mathrm{cm}^{2}$ and (b) $F=2.4 \mathrm{~J} / \mathrm{cm}^{2}$. The $\mathrm{Ca} / \mathrm{P}$ ratio does not show a clear spatial distribution for different values of spot area or $F$. The results shown in Figs. 2 and 3 indicate that the desorbed chemical species from the target exhibit a uniform chemical composition that is independent of both the spot area and $F$; their spatial distribution depends on the spot area. Spot area and $F$, however, dramatically affect the absolute value of the $\mathrm{Ca} / \mathrm{P}$ ratio. Because the sample prepared in this study does not exhibit a spatial $\mathrm{Ca} / \mathrm{P}$ ratio distribution, we define a net $\mathrm{Ca} / \mathrm{P}$ ratio for each sample by averaging the $\mathrm{Ca} / \mathrm{P}$ ratios

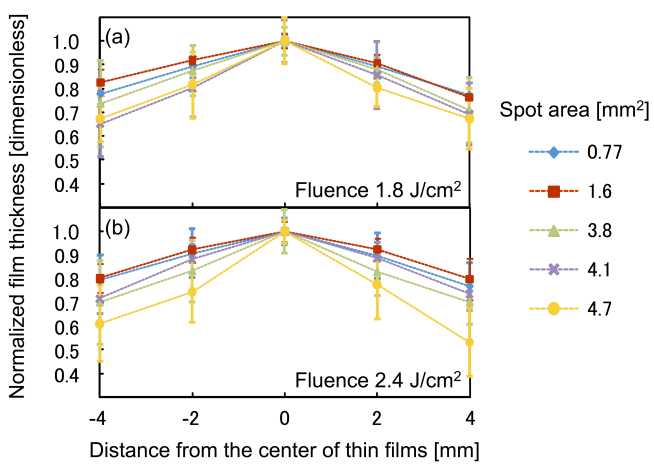

Fig. 2. Dependence of the spatial thickness distribution of HA thin films on spot area for $F=$ (a) $1.8 \mathrm{~J} / \mathrm{cm}^{2}$ and (b) $2.4 \mathrm{~J} / \mathrm{cm}^{2}$. A larger spot area is correlated with a narrower thickness distribution. 


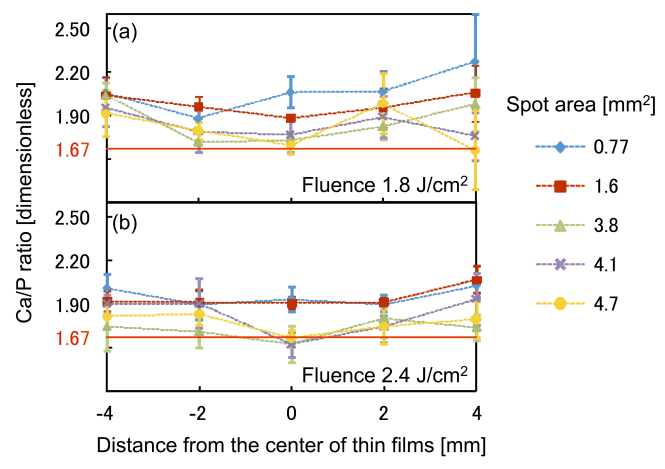

Fig. 3. Dependence of spatial $\mathrm{Ca} / \mathrm{P}$ ratio distribution of HA thin film on spot area assuming fluence of (a) $1.8 \mathrm{~J} / \mathrm{cm}^{2}$ and (b) $2.4 \mathrm{~J} / \mathrm{cm}^{2}$. The $\mathrm{Ca} / \mathrm{P}$ ratio does not show the spatial distribution. This result suggests that the HA thin film has a uniform $\mathrm{Ca} / \mathrm{P}$ ratio that is independent of the spot area.

measured at five points, as shown in Fig. 3. The net $\mathrm{Ca} / \mathrm{P}$ ratios are then summarized as a function of the spot area in Fig. 4 for $F=1.8 \mathrm{~J} / \mathrm{cm}^{2}$ and $2.4 \mathrm{~J} / \mathrm{cm}^{2}$. We found that the spot area changes the chemical composition dramatically for the thin films that were prepared via PLD. While the effect of the spot area on the chemical composition has been reported elsewhere, the effect of the spot area was not strictly separated from the variations in $F$, as discussed in Section 1 . That is, $F$ is changed simultaneously with the spot area, as reported in the literature ${ }^{22,23}$. Based on Fig. 4, we also see that a larger $F$ value with a constant spot area reduces the $\mathrm{Ca} / \mathrm{P}$ ratio. The best parameters for the $\mathrm{Ca} / \mathrm{P}$ ratio of $1.72 \pm$ 0.13 are a spot area of $3.8 \mathrm{~mm}^{2}$ and $F=2.4 \mathrm{~J} / \mathrm{cm}^{2}$.

We have succeeded in improving the $\mathrm{Ca} / \mathrm{P}$ ratios of HA thin films. The physics behind the way in which spot area controls chemical composition is not yet understood, although the importance of the spot area is proposed without doubt for the first time in this study. The results given here suggest that the chemical compositions of thin films prepared via PLD are not always close to that of the target. Even so, PLD is generally considered to be one of the most useful techniques for preparation of thin films with well-defined chemical compositions that are similar to that of the target. Further precise control of the chemical composition requires us to reveal the physics of how the spot area controls the chemical composition. To study the physics of this process, the spatial distribution of the laser energy should be homogenized because the chemical composition must vary with the fluence. In this study, $F$ corresponds to the spatial average of the energy density. Such a definition of $F$ will be too imprecise to enable discussion of the process physics. Chemical species are desorbed with different $\mathrm{Ca} / \mathrm{P}$ ratios that depend on the fluence from each spatial position, while the $\mathrm{Ca} / \mathrm{P}$ ratio measured in the study is an integrated average for chemical species desorbed from every position in the spot area. The $\mathrm{Ca} / \mathrm{P}$ ratio dependence, not on the average, but on the uniform and precise fluence should be measured to avoid measurement of an integrated average of the different $\mathrm{Ca} / \mathrm{P}$ ratios from

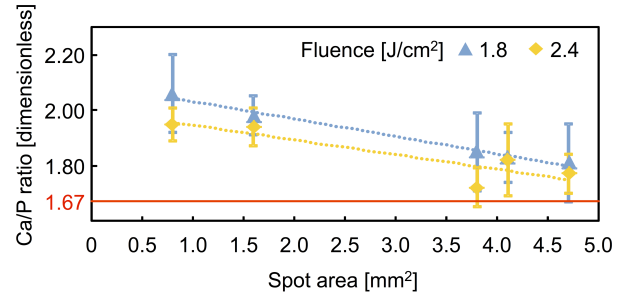

Fig. 4. Dependence of $\mathrm{HA}$ thin film $\mathrm{Ca} / \mathrm{P}$ ratio on spot area. The spot area changes the $\mathrm{Ca} / \mathrm{P}$ ratio significantly; larger spot areas improve the $\mathrm{Ca} / \mathrm{P}$ ratio for both fluences studied here. The best parameters are spot area $=3.8 \mathrm{~mm}^{2}$ and $F=2.4$ $\mathrm{J} / \mathrm{cm}^{2}$.

positions with different fluences in the spot area. As a next step, we intend to investigate the physics based on the measured dependence of the $\mathrm{Ca} / \mathrm{P}$ ratio on the precise and uniform fluence.

\section{CONCLUSION}

We have aimed to improve the $\mathrm{Ca} / \mathrm{P}$ ratios of $\mathrm{HA}$ thin films prepared via PLD. The chemical composition, and particularly the $\mathrm{Ca} / \mathrm{P}$ ratio, is critical to the biodegradability of HA for use in high-quality medical and dental devices. We varied the spot area of the laser pulse independently of the laser fluence. Similar studies have previously been conducted, but these studies did not change the spot area and the fluence independently. Because the fluence is generally believed to be one of the most important parameters in the ablation process, the effect of the spot area was not evaluated properly in these previous studies. To maintain a constant fluence as the spot area was varied, the total energy irradiated on the target surface was adjusted relative to the spot area, which was measured in advance. We found that the spot area causes changes in the chemical compositions of the thin films prepared via PLD, independently of the fluence. We achieved a $\mathrm{Ca} / \mathrm{P}$ ratio of $1.72 \pm 0.13$ with a spot area and fluence of $3.8 \mathrm{~mm}^{2}$ and $2.4 \mathrm{~J} / \mathrm{cm}^{2}$, respectively, and this ratio is close to the stoichiometric HA value. The importance of the spot area is reported here for the first time; understanding of the physics behind this result will be the next step.

\section{ACKNOWLEDGEMENTS}

This work was supported in part by JSPS KAKENHI Grant-in-Aid for Scientific Research (C) (grant number 24560019) and by a grant from the Strategic Research Foundation Grant-aided Project for Private Universities from the Ministry of Education, Culture, Sport, Science, and Technology, Japan (MEXT), 2013-2017 (grant number S1311045).

\section{References}

[1] R. G. T. Geesink, K. de Groot, and C. P. A. T. Klein, Clin. Orthop. Relat. Res., 225, 147-170 (1987).

[2] C. P. A. T. Klein, P. Patka, H. B. M. van der Lubbe, J. G. C. Wolke, and K. de Groot, J. Biomed. Mater. Res., 25, 53-65 (1991).

[3] R. McPherson, N. Gane, and T. J. Bastow, J. Mater. 
Sci. - Mater. Med., 6, 327-334 (1995).

[4] W.-Q. Yan, T. Nakamura, K. Kawanabe, S. Nishiguchi, M. Oka, and T. Kokubo, Biomaterials, 18, 1185-1190 (1997).

[5] A. Stoch, A. Brożek, G. Kmita, J. Stoch, W. Jastrzębski, and A. Rakowska, J. Mol. Struct., 596, 191-200 (2001).

[6] J. L. Ong, L. C. Lucas, W. R. Lacefield, and E. D. Rigney, Biomaterials, 13, 249-254 (1992).

[7] M. Yoshinari, T. Hayakawa, J. G. Wolke, K. Nemoto, and J. A. Jansen, J. Biomed. Mater. Res., 37, 60-67 (1997).

[8] A. M. Ektessabi and H. Kimura, Thin Solid Films, 270, 335-340 (1995).

[9] A. M. Ektessabi, Nucl. Instrum. Methods Phys. Res., Sect. B, 127-128, 1008-1014 (1997).

[10] H. Zeng, K. K. Chittur, and W. R. Lacefield, Biomaterials, 20, 443-451 (1999).

[11] K. Yamashita, T. Arashi, K. Kitagaki, S. Yamada, T. Umegaki, and K. Ogawa, J. Am. Ceram. Soc., 77, 2401-2407 (1994)

[12] J. G. C. Wolke, K. van Dijk, H. G. Schaeken, K. de Groot, and J. A. Jansen, J. Biomed. Mater. Res., 28, 1477-1484 (1994).

[13] L. Torrisi and R. Setola, Thin Solid Films, 227, 32-36 (1993).

[14] S. Hontsu, M. Nakamori, H. Tabata, J. Ishii, and T. Kawai, Jpn. J. Appl. Phys., 35, L1208-L1210 (1996).

[15] B. C. Wang, T. M. Lee, E. Chang, and C. Y. Yang, J. Biomed. Mater. Res., 27, 1315-1327 (1993).

[16] K. A. Gross and C. C. Berndt, J. Biomed. Mater. Res., 39, 580-587 (1998).

[17] M. Okazaki, T. Aoba, Y. Doi, J. Takahashi, and Y. Moriwaki, J. Osaka Univ. Dent. Sch., 19, 87-93 (1979).

[18] M. Kusunoki, M. Kawasima, H. Nishikawa, K. Morimoto, T. Hayami, S. Hontsu, and T. Kawai, Jpn. J. Appl. Phys., 44, L326-L327 (2005).

[19] Y. Hashimoto, M. Kusunoki, R. Hatanaka, K. Hamano, H. Nishikawa, Y. Hosoi, S. Hontsu, and M. Nakamura, Adv. Sci. Technol., 49, 282-289 (2006).

[20] H. Nishikawa, R. Hatanaka, M. Kusunoki, T. Hayami, and S. Hontsu, Appl. Phys. Express, 1, 088001-1-088001-3 (2008).

[21] Y. Sakoishi, R. Iguchi, H. Nishikawa, S. Hontsu, T. Hayami, and M. Kusunoki, Appl. Phys. Express, 6, 115501-1-115501-3 (2013).

[22] T. Ohnishi, M. Lippmaa, T. Yamamoto, S. Meguro, and H. Koinuma, Appl. Phys. Lett., 27, 241919-1-241919-3 (2005).

[23] J. H. Song, T. Susaki, and H. Y. Hwang, Adv. Mater., 20, 2528-2532 (2008).

(Received December 15, 2014; Accepted February 23, 2015) 